\title{
Ultra-high Dimensional Variable Screening via Density Weighted Variance
}

\author{
Jingke Zhou and Yingzhen Chen*
}

Statistics and Econometrics School of Business, Ningbo University, Ningbo, Zhejiang, P.R. China

\begin{abstract}
Density Weighted Variance (DWV), a novel model-free feature screening criterion is proposed for mean regression with ultrahigh-dimensional covariates. Compared with existing model free screening criteria, DWV criterion possesses faster convergence rate for inactive co-varieties and is as same convergence rate as most existing variable screening procedures for active covariates. Furthermore, DWV criterion is extended to quintile regression and multiple response regression setting. Finally, numerical simulations and a real data analysis are conducted to show the finite sample performance of the proposed methods.
\end{abstract}

Keywords: Model-free variable screening; Density weighted variance; Ultra-high dimensional data analysis

\section{Introduction}

High dimensional data analysis have been stirring statistical area as data collection becoming more and more convenient. However, many conventional statistical methods couldn't be used directly to deal with high dimensional data, variable selection and dimension reduction are two main techniques to bridge the gap between conventional statistical methods and modern high dimensional data. In this paper, we restrict our attentions to high dimensional variable selection procedures.

Fan and Lv [1] provided a selective overview on existing variable selection methods before 2008. Fan and Lv [2] proposed marginal correlation coefficient screening for ultra-high dimensional linear regression model. After the seminal paper, many other marginal criteria are proposed for linear regression model, generalized linear model and other parametric or nonpara metric regression models, such as, Wang [3], Fan and Song [4], Fan et al. [5], Li et al. [6], and so on. Recently, model-free variable screening procedures received some attention to improve the performances when the model couldn't be specified. Based on distance correlation measure [7], Li et al. [8], proposed DC-SIS procedure. For mean regression model, Shao and Zhang [9] modified the definition of distance correlation and proposed MDC-SIS variable screening method. For discriminant analysis or discrete response regression model, Cui et al. [10] proposed MV-SIS by comparing empirical distribution function.

The purpose of this paper is to propose a new model-free variable screening criterion-Density Weighted Variance(DWV, hereafter) for mean regression model and to extend it to other general regression models which model functionals of conditional distribution of response variable $\mathrm{Y}$ given covariates $\mathrm{X}$. Compared with existing model free screening criteria, DWV criterion has faster convergence rate for inactive covariates and has same convergence rate as most existing variable screening procedures for active covariates. Furthermore, the prosed procedure imposes less constraints on response variable, it can be used for univariate or multivariate, discrete or continuous response variable.

The remainder of this paper is organized as follows. In section 2, we develop the DWV-SIS for mean regression. In section 3, we extend the proposed variable screening procedure to quantile regression. In section 4, multiple response regression model was considered. Numerical simulations and real data analysis are conducted in section 5 to show the finite sample performance of the proposed methods. All technical proofs are given in Appendix.

\section{DWV Criterion for Mean Regression}

This section is divided into three parts. We present some preliminaries on smooth test statistic and the motivation of DWV criterion in subsection 1. Sequentially, we develop DWV-SIS method for mean regression with continuous covariate variables in subsection 2. Finally, we consider mean regression with mixed discrete and continuous covariate variables in subsection 3 .

\section{Preliminaries and motivation}

Consider a parametric mean regression

$$
\mathrm{E}(\mathrm{Y} \mid \mathrm{X})=\mathrm{g}(\mathrm{X}, \theta)
$$

where $\mathrm{g}(\cdot)$ is a known function, and $\theta \in \Theta$ is unknown parameter. For a given i.i.d. data, $\left\{\left(x_{i}, y_{i}\right), i=1,2, \ldots, n\right\}$, to test the goodness of fit of the parametric model (1), let $\varepsilon=Y-g\left(X, \theta_{0}\right)$, where ${ }_{0}$ is the projection of $Y$ onto parametric family $\{g(X, \theta): \theta \in \Theta\}$, that is,

$$
\theta_{0}=\arg \min _{\theta \in \Theta} E[Y-g(X, \theta)]^{2}
$$

Zheng noted that, under the null hypothesis $\mathrm{E}(\mathrm{Y} \mid \mathrm{X})=\mathrm{g}(\mathrm{X}, \theta 0)$, $\mathrm{E}(\varepsilon \mid \mathrm{X})=0$ and

$$
E[\varepsilon E(\varepsilon \mid X) f(X)]=E\left[E^{2}(\varepsilon \mid X) f(X)\right]=0
$$

whereas $E[\varepsilon E(\varepsilon \mid X) f(X)]>0$ under alternative hypothesis. By estimating the conditional expectation and density function using conventional kernel smooth estimator, Zheng [11] proposed the following test statistic:

$$
\hat{V}_{n}=\frac{1}{n(n-1)} \sum_{i \neq j} K_{h}\left(x_{i}, x_{j}\right)\left[y_{i}-g\left(x_{i}, \hat{\theta}\right)\right]\left[y_{j}-g\left(x_{j}, \hat{\theta}\right)\right]
$$

where $\hat{\theta}$ is a $\sqrt{ }$ n-consistent estimator of $\theta_{0}$. The $\mathrm{U}$-statistic structure endues the test statistic several appealing properties, detailed discussion will be omitted here and readers who are interested in can refer Zheng.

*Corresponding author: Yingzhen Chen, Ningbo University, Ningbo, Zhejiang, P.R. China, Tel: 868367441118; E-mail: chenyingzhen@nbu.edu.cn

Received May 15, 2018; Accepted June 13, 2018; Published June 18, 2018

Citation: Zhou J, Chen Y (2018) Ultra-high Dimensional Variable Screening via Density Weighted Variance. J Biom Biostat 9: 401. doi: 10.4172/21556180.1000401

Copyright: ( 2018 Zhou J, et al. This is an open-access article distributed under the terms of the Creative Commons Attribution License, which permits unrestricted use, distribution, and reproduction in any medium, provided the original author and source are credited. 
However, the curse of dimensionality still befalls it because of the nonparametric estimator nature.

Another paper which motivate our work is Shao and Zhang [9]. Inspired by the definition of Distance Correlation (DC) [7], they proposed a new correlation measure, Martingale Difference Correlation $(\mathrm{MDC})$, for mean regression based on $\mathrm{E}(\mathrm{Y} \mid \mathrm{X})=\mathrm{E}(\mathrm{Y})$ almost surely. Compared with traditional Pearson correlation, MDC can measure nonlinearity correlation, on the other hand, $\mathrm{DC}(\mathrm{X}, \mathrm{Y})=0$ is equivalent to $\mathrm{X}$ and $\mathrm{Y}$ are independent, which is a bit stronger for mean regression whereas MDC is enough.

The DWV criterion is also based on $\mathrm{E}(\mathrm{Y} \mid \mathrm{X})=\mathrm{E}(\mathrm{Y})$ for mean regression. Let $\varepsilon=\mathrm{Y}-\mathrm{E}(\mathrm{Y})$, if somecovariate variable $\mathrm{Xk}$ has no contribution to the mean of $\mathrm{Y}$, i.e. $\mathrm{E}(\mathrm{Y} \mid \mathrm{Xk})=\mathrm{E}(\mathrm{Y})$ almost surely, then, $\mathrm{E}[\varepsilon \mathrm{E}(\varepsilon \mid \mathrm{Xk}) \mathrm{f}(\mathrm{Xk})]=0$, otherwise, $\mathrm{E}[\varepsilon \mathrm{E}(\varepsilon \mid \mathrm{Xk}) \mathrm{f}(\mathrm{Xk})]>0$. The criterion can be estimated by the following U-statistic

$$
\hat{V}_{n}=\frac{1}{n(n-1)} \sum_{i \neq j} K_{h}\left(x_{k, i}, x_{k, j}\right)\left[y_{i}-\bar{y}\right]\left[y_{j}-\bar{y}\right]
$$

What's more, the curse of dimensionality can be avoided since we calculate the criterion for all covariate one by one in variable screening, while the appealing properties of $\mathrm{U}$-statistics are preserved.

\section{DWV-SIS for continuous covariates}

Let $\mathrm{Y}$ be response variable and $\{\mathrm{Xk}, \mathrm{k}=1,2, \ldots \mathrm{p}\}$ are continuous covariate variables. Similar to Shao and Zhang [9], we define active covariate variables set as

$\mathrm{A}=\{k: \mathrm{E}(\mathrm{Y} \mid \mathrm{X} k)$ depends on $\mathrm{X} k\}$,

and inactive covariate variables set

$I=\{k: \mathrm{E}(\mathrm{Y} \mid \mathrm{X} k)$ does not depend on $\mathrm{X} k\}$.

For ease of presentation, we write:

$\mathrm{V} k=\mathrm{E}\left\{[\mathrm{E}(\mathrm{Y} \mid \mathrm{X} k)-\mathrm{E}(\mathrm{Y})]^{2} \mathrm{f}(\mathrm{X} k)\right\}$.

Obviously, from population view of point, we have, for any $\mathrm{k} \in \mathrm{I}$, $\mathrm{V} k=0$, while $\mathrm{V} k>0$ for any $\mathrm{k} \in \mathrm{A}$. As same as most variable screening procedure, we select the covariate variables with large $\hat{V}_{k}$ as active covariate variables, that is,

$$
\hat{\mathcal{A}}=\left\{k: \hat{V}_{k} \geq \alpha\right\}
$$

where $\alpha>0$ is preassigned threshold. It is more convenient to rank the DWV criterion and select the largest $d$ covariate variables as active covariate variables in real data application.

Next, we study the theoretical properties of the proposed DWV-SIS procedure. The following assumptions and conditions are imposed to facilitate the technical proofs, although they may not be the weakest ones.

(A.1) There are positive constants $\mathrm{c}>0$ and $0 \leq \kappa<1 / 2$ such that,

$$
\left.\inf _{k \in \mathcal{A}} E\left[\left(\mathrm{EY} \mid \mathrm{X}_{k}\right)-\mathrm{E}(Y)\right)^{2} \int f\left(X_{k}\right)\right] \geq 2 c n^{-k}
$$

(A.2) The response variable $Y$ satisfy the sub-exponential tail probability, that is, there exists a positive constant $\mathrm{s} 0$ such that for all $0<s \leq 2 s_{0}, E\left[\exp \left(s Y^{2}\right)\right]<\infty$.

This assumption is identical to condition (C.1) in Li et al. [8], and under this assumption, we have, $\mathrm{E}\left\{\exp [\mathrm{s}(Y-E(Y))]^{2}\right\}<\infty$.

(A.3) For all $1 \leq k \leq \mathrm{p}$, the density function of $X k, \mathrm{f}(x k)$ is twice continuous differentiable, both $\mathrm{f}(\mathrm{x} k)$ and its first order derivative are uniformly bounded.
This assumption is similar to the first part of Assumption 1 in Zheng [11], and our assumption is just make assumption on marginal density function whereas Zheng [11] make assumption on joint density function which can imply our assumption.

(A.4) For all $k \in \mathrm{A}, \mathrm{E}\left(Y^{4} \mid \mathrm{X}_{k}=\mathrm{x}_{k}\right)$ is twice continuously differentiable and bounded by a measurable function $\mathrm{b}(\mathrm{xk})$ such that $\mathrm{E}\left[b^{2} X_{k}\right]<\infty$.

This assumption is similar to the second part of Assumption 1 in Zheng [11].

(C.1) Kernel function $\mathrm{K}(u)$ is symmetric and satisfies that, $\int u^{2} K(u) d u<\infty, k_{h}(u)=\frac{1}{h} K\left(\frac{u}{h}\right)$ is uniformly bounded respect to $u$ and $h>0$. That is, there is a positive constant $M_{1}>0$ such that, $\sup _{\mathrm{h}>0 . u}$ $\mathrm{K}_{\underline{h}}(u) \leq M_{1}<\infty$. Moreover, the bandwidth h satisfies that $h \rightarrow 0$ and $n h$ $\rightarrow \infty$ as $n \rightarrow \infty$.

For bounded support kernel, this condition is equivalent to $K(u)$ is bounded which is an all pervading condition in nonparametric kernel literatures. While for unbounded support kernel, this condition requires the convergence rate of $K\left(\frac{u}{h}\right)$ is not slower than that of $h$, and the habitual Gaussian kernel is satisfied.

First, we present the convergence results proved in Zheng [11],

Theorem 1: Under assumptions (A.2)-(A.4) and Condition (C.1), for any $k \in I$, we have, $\hat{V}_{k}=V_{k}+O_{p}\left(n^{-1} h^{-1 / 2}\right)$, and for any $\mathrm{k} \in \mathrm{A}$, we have, $\hat{V}_{k}=V_{k}+O_{p}\left(n^{-1 / 2}\right)$.

From variable selection viewpoint, we have the following sure screening property.

Theorem 2. Under assumptions (A.2)-(A.4) and Condition (C.1) for any $0<\gamma<1 / 2-\kappa$, there exists a constants $c_{1}>0$, such that,

$$
P \gamma\left(\sup _{1 \leq k \leq p}\left|\hat{V}_{n, k}-V_{k}\right| \geq O\left(p\left[2 \exp \left\{-\mathrm{n}^{1-2 \gamma-2 k}\right\}+\mathrm{nc}_{1} \exp \left\{-\mathrm{sn}^{\gamma}\right\}\right]\right)\right)
$$

By assumptions (A.1) and theorem 2, we can take $\alpha=c n^{-k}$ in (5), then,

$$
\operatorname{P\gamma }(\mathcal{A} \subset \hat{\mathcal{A}}) \geq 1-O\left(|\mathcal{A}|\left[2 \exp \left\{-\mathrm{n}^{1-2 \gamma-2 k}\right\}+\mathrm{nc}_{1} \exp \left\{-\mathrm{sn}^{\gamma}\right\}\right]\right)
$$

where $|\mathrm{A}|$ is the cardinality of $\mathrm{A}$.

It is worth mentioning that Fan et al. [12] provided a nonasymptotic bound for sure independence screening procedure based on a variant of SIS by assuming exchangeability condition, which is also hold for our DWV-SIS procedure since the proof is independent with screening criterion, and we restate the result here, more details can be referred in Fan et al. [12].

(A.5) (Exchangeability condition) - Let $r \in N$, the set of natural number. We say the model satisfies the exchangeability condition at level $r$ if the set of random vectors:

$\left\{\left(Y, X \mathrm{~A}, X_{j 1}, X_{j 2}, \cdots, X_{j r}\right): \mathrm{j}_{1}, \mathrm{j}_{2}, \cdots, \mathrm{jr}\right.$ are distinct elements of $\left.\mathrm{A}^{\mathrm{c}}\right\}$ is exchangeable.

Theorem 3: Under assumptions (A.1)-(A.4), Condition (C.1), and there is $r \in N$ such that assumption (A.5) hold, then

$$
P \gamma\left(\left|\hat{\mathcal{A}} \cap \mathcal{A}^{C}\right|>\gamma\right) \leq \frac{\left(C_{d}^{\gamma}\right)^{2}}{C_{p-|\mathcal{A}|}^{\gamma}} \leq \frac{1}{\gamma}\left(\frac{d^{2}}{p-|\mathcal{A}|}\right) \gamma
$$

\section{DWV-SIS for discrete covariates}

To deal with nonparametric kernel estimation with categorical 
covariate variables, Aitchison and Aitken [13] proposed binomial kernel as follows

$$
B \lambda(x i, x j)=\lambda^{1-d(x i, x j)}(1-\lambda)^{d(x i, x j)}
$$

where $1 / 2 \leq \lambda \leq 1$ is smooth parameter, a habitual definition of $d(x$, $y$ ) is 0 when $x=y$, otherwise 1 if $x \neq y$. Subsequently, Hsiao et al. [14] suggested a variation which can also treat ordinal data as follows

$$
B \lambda(x i, x j)=\lambda^{d(x i, x j)}
$$

where smooth parameter $\lambda \in[0,1]$. In this paper, the variational binomial kernel (10) is used to handle discretecovariate variable. Thus, our DWV criterion for discretecovariate variable is

$$
\hat{V}_{k}^{(\mathcal{D})}=\frac{1}{n(\mathrm{n}-1)} \sum_{i \neq j} B_{\lambda}\left(x_{k, i}, x_{k, j}\right)\left[y_{i}-\bar{y}\right]\left[y_{j}-\bar{y}\right]
$$

and we have the following convergence result.

Theorem 4: Under assumptions (A.2) and Condition (C.1), for any $k \in I$, we have, $\hat{V}_{k}=V_{k}+O_{p}\left(\mathrm{n}^{-1}\right)$, and for any $\mathrm{k} \in \mathrm{A}$, we have, $\hat{V}_{k}=V_{k}+O_{p}\left(\mathrm{n}^{-1 / 2}\right)$

From variable selection viewpoint, we have the following sure screening property.

Theorem 5: Under assumptions (A.2) and Condition (C.1), for any $0<\gamma<1 / 2-\kappa$, there exists a constants $\mathrm{c}_{1}>0$, such that,

$$
\left.P \gamma\left(\sup _{1 \leq k \leq p}\left|\hat{V}_{n, k}-V_{k}\right| \geq c n^{-k}\right) \leq O\left(p\left[2 \exp \left\{-\mathrm{n}^{1-2 \gamma-2 k}\right\}+\mathrm{nc}_{1} \exp \left\{-\mathrm{sn}^{\gamma}\right\}\right]\right)\right)
$$
then,

By assumptions (A.1) and theorem 4, we can take $\alpha=\mathrm{cn}-\kappa$ in (5),

$$
P \gamma(\mid \mathcal{A} \cap \hat{A}) \geq 1-O\left(|\mathcal{A}|\left[2 \exp \left\{-\mathrm{n}^{1-2 \gamma-2 k}\right\}+\mathrm{nc}_{1} \exp \left\{-\mathrm{sn}^{\gamma}\right\}\right]\right),
$$

where $|\mathrm{A}|$ is the cardinality of A.

\section{DWV-SIS for Multiple Response Regression}

We have to consider multiple response regression model for some situations, for instance, we have to introduce several dummy variables when the original response variable $\mathrm{Y}$ take multi-categorical nominal data. On the other hand, multiple response joint modeling may improve the performance when the components of $Y$ are dependent each other. For multiple response regression model, denote $\lambda \max (\cdot)$ be the largest eigen value of a matrix, we propose the following DWV criteria.

$$
\hat{V}_{k}=\lambda_{\max }\left\{\frac{1}{n(n-1)} \sum_{i \neq j} k_{\lambda}\left(x_{k, i}, x_{k, j}\right)\left[y_{i}-\bar{y}\right]\left[y_{j}-\bar{y}\right]^{T}\right\}
$$

for $k \in \mathrm{C}$ and

$$
\hat{V}_{k}=\lambda_{\max }\left\{\frac{1}{n(n-1)} \sum_{i \neq j} B_{\lambda}\left(x_{k, i}, x_{k, j}\right)\left[y_{i}-\bar{y}\right]\left[y_{j}-\bar{y}\right]^{T}\right\}
$$

for $\mathrm{k} \in \mathrm{D}$.

To study the asymptotical properties, we make the following assumptions:

(A.5) There are positive constants $c>0$ and $0 \leq \kappa<1 / 2$ such that,

$$
\left.\inf _{k \in \mathcal{A}} \| E\left[\mathrm{E}\left(\mathrm{Y} \mid \mathrm{X}_{k}\right)-\mathrm{E}(\mathrm{Y})\right)\left(\mathrm{E}\left(\mathrm{Y} \mid \mathrm{X}_{k}\right)-\mathrm{E}(\mathrm{Y})\right)^{\mathrm{T}} f\left(X_{k}\right)\right] \| \geq 2 c n^{-k} .
$$

(A.6) The response variable $\mathrm{Y}$ satisfy the sub-exponential tail probability, that is, there exists a positive constant s0 such that for all $0<\mathrm{s} \leq 2 s_{0}, \mathrm{E}\left[\exp \left(\mathrm{s}\left\|\mathrm{YY}^{\mathrm{T}}\right\|\right)\right]<\infty$.
(A.7) For all $k \in \mathrm{A}, \mathrm{E}\left(\mathrm{Y}^{4} \mid \mathrm{X}_{\mathrm{k}}=\mathrm{x}_{\mathrm{k}}\right)$ is twice continuously differentiable and bounded by a measurable function $b(x k)$ such that $E\left[b^{2} X_{k}\right]<\infty$.

Before stating the theoretical results, we restate the following lemma first, which is extracted from corollary (3.2.6) of Bhatia [15]. Then,

Lemma 1 (Weyl's inequality): Let A and B be Hermitian matrices.

$$
\max _{j}\left|\lambda_{j}(A)-\lambda_{j}(\mathrm{~B})\right| \leq\|A-B\|
$$

where $\|\cdot\|$ is operator norm of a matrix, that is, the largest eigenvalues. By the equivalence between matrix norms, there is a constant $c>0$, such that,

$$
\max _{j}\left|\lambda_{j}(A)-\lambda_{j}(\mathrm{~B})\right| \leq c\|A-B\|_{F}
$$

where $\|\cdot\|_{\mathrm{F}}$ is Frobenius norm of a matrix.

Theorem 6: Under assumptions (A.3), (A.6), (A.7) and Condition (C.1), for any $\mathrm{k} \in \mathrm{I}$, we have, $\hat{V}_{k}=V_{k}+O_{p}\left(n^{-1} h^{-1 / 2}\right)$ and for any $\mathrm{k} \in \mathrm{A}$, we have, $\mathrm{V}^{\wedge} \mathrm{k}=\mathrm{Vk}+\mathrm{OP}\left(\mathrm{n}^{-\frac{1}{2}}\right)$

From variable selection viewpoint, we have the following sure screening property.

Theorem 7. Under assumptions (A.3), (A.6), (A.7) and Condition (C.1), for any $0<\gamma<1 / 2-\kappa$, there exists a constants $\mathrm{c} 1>0$, such that,

$$
\left.P \gamma\left(\sup _{1 \leq k \leq p}\left|\hat{V}_{n, k}-V_{k}\right| \geq c n^{-k}\right) \leq O\left(p\left[2 \exp \left\{-\mathrm{n}^{1-2 \gamma-2 k}\right\}+\mathrm{nc}_{1} \exp \left\{-\mathrm{sn}^{\gamma}\right\}\right]\right)\right)_{k} \text {. }
$$
then,

By assumptions (A.5) and theorem 7, we can take $\alpha=\mathrm{cn}^{\alpha k}$ in (5),

$$
\operatorname{P\gamma }(\mid \mathcal{A} \cap \hat{\mathcal{A}}) \geq 1-O\left(|\mathcal{A}|\left[2 \exp \left\{-\mathrm{n}^{1-2 \gamma-2 k}\right\}+\mathrm{nc}_{1} \exp \left\{-\mathrm{sn}^{\gamma}\right\}\right]\right),
$$

where $|\mathrm{A}|$ is the cardinality of $\mathrm{A}$.

\section{DWV Criterion for Quantile Regression}

In this section, we extend the DWV-SIS procedure to quantile regression setting by means of a transformation. Consider the following quantile regression model

$$
\mathrm{Q} \tau(\mathrm{Y} \mid \mathrm{X})=\mathrm{g}(\mathrm{X})
$$

If some covariate variable $\mathrm{X}_{\mathrm{k}}$ has no contribution to the $\tau$-quantile of $\mathrm{Y}$, then $\mathrm{Q} \tau\left(\mathrm{Y} \mid \mathrm{X}_{\mathrm{k}}\right)=\mathrm{Q} \tau(\mathrm{Y})$, otherwise, $\mathrm{Q} \tau\left(\mathrm{Y} \mid \mathrm{X}_{\mathrm{k}}\right)=\mathrm{Q}\left(\mathrm{x}_{\mathrm{k}}\right)$. Note that, for continuous response variable $\mathrm{Y}$, we have,

$\mathrm{E}[1(\mathrm{Y} \leq \mathrm{Q} \tau)]=\operatorname{Pr}(\mathrm{Y} \leq \mathrm{Q} \tau))=\tau$.

Thus, the quantile regression model (20) is equivalent to $\mathrm{E}(1(\mathrm{Y} \leq \mathrm{g}(\mathrm{X})) \mid \mathrm{X})=\tau$.

Based on this fact, we propose DWV criterion for quantile regression as follows

$$
\mathrm{Vk}=\mathrm{E}\left\{[\mathrm{E}(1(\mathrm{Y} \leq \mathrm{Q} \tau(\mathrm{Y}))-\tau \mid \mathrm{X})]^{2} \mathrm{f}\left(\mathrm{X}_{\mathrm{k}}\right)\right\} \cdot
$$

Obviously, $\mathrm{Vk}=0$ for $\mathrm{k} \in \mathrm{I}$ and $\mathrm{Vk}>0$ for $\mathrm{k} \in \mathrm{A}$.

For a given i.i.d. sample data, we can estimated the criterion as follows

$$
\left.\hat{V}_{k}=\frac{1}{n(\mathrm{n}-1)} \sum_{i \neq j} K_{h}\left(x_{k, i}, x_{k, j}\right)\left[1\left(y_{i} \leq \hat{Q}_{\tau}(Y)\right)-\tau\right]\left[1 y_{j} \leq \hat{Q}_{\tau}(Y)\right)-\tau\right]
$$


for $\mathrm{k} \in \mathrm{C}$,

and

$$
\hat{V}_{k}=\frac{1}{n(\mathrm{n}-1)} \sum_{i \neq j} B_{h}\left(x_{k, i}, x_{k, j}\right)\left[1\left(y_{i} \leq \hat{Q}_{\tau}(Y)\right)-\tau\right]\left[1\left(y_{j} \leq \hat{Q}_{\tau}(\mathrm{Y})\right)-\tau\right]
$$

for $\mathrm{k} \in \mathrm{D}$.

Furthermore, we can develop DWV criterion for general regression which model a general functional of the conditional distribution $\mathrm{Y}$ given $\mathrm{X}$. Let $\tau \sim \mathrm{U}(0,1)$, we propose DWV criterion for regression as follow

$$
\mathrm{Vk}=\mathrm{E} \tau\left\{\mathrm{EX}\left\{[\mathrm{EY}(1(\mathrm{Y} \leq \mathrm{Q} \tau(\mathrm{Y}))-\tau \mid \mathrm{X})]^{2} \mathrm{f}\left(\mathrm{X}_{\mathrm{k}}\right)\right\}\right\} .
$$

The sure screening property can be proved similarly.

\section{Simulation Study}

\section{Determination of bandwidth}

Bandwidth selection is a critical procedure for nonparametric estimation, DWV-SIS is no exception. Most of existing methods depend on the accuracy measure of regression model based on selected active covariates. In this paper, inspired by stable selection method, we propose a bootstrap strategy which doesn't rely on the regression model. Denote $\left\{h_{s}\right\}_{s=1}^{L}$ be the candidate bandwidth.

Step 1: Drawing bootstrap sample $\left\{x^{b}, y^{b}\right\}_{b=1}^{B}$ from sample data $\left\{\left(x_{i}, y_{i}\right)\right\}_{i=1}^{n}$, and selecting the active variables for each $\left\{h_{s}\right\}_{s=1}^{L}$, then we can obtain $\mathrm{L} \times \mathrm{B}$ active covariate variables set $\hat{\mathcal{A}}_{b, s}$.

Step 2: For each covariate, count the number that it be selected by some active covariate variables set $\hat{\mathcal{A}}_{b, s}$, and denote the covariates whose selected number is larger than $[\alpha \mathrm{LB}]$ as estimated "true" active covariate $\hat{A}$.

Step 3: The optimal bandwidth hopt is determined by the following optimization $\arg \min _{h_{s}}\left\{\frac{1}{B} \sum_{b=1}^{B}\left\|\hat{A}-\hat{A}_{b, s}\right\|\right\}$ that is, the one with smallest average false positive number. In our simulations, we set $[\alpha \mathrm{LB}]=[\mathrm{n} /$ $\log (\mathrm{n})]$ and $\mathrm{B}=50$.

$\mathrm{h}=(\mathrm{h} 1, \mathrm{~h} 2, \ldots, \mathrm{hp})$ where $\mathrm{h}_{k}=c n^{-4 / 5} \sigma_{X_{k}}$ for continuous covariate variable and $h_{k}=c n^{-1 / 5} \sigma_{X_{k}}$ for discrete covariate variable, $\mathrm{c}=0.2,0.4, \ldots, 10$.

\section{Numerical simulation}

In this section, we assess the finite sample performance of the proposed method via Monte Carlo simulation. We set $\mathrm{p}$ to be $1000, \mathrm{~d}$ to be $[n / \log (n)]$. All the simulation results are based on 500 replications. We consider the linear model in the Examples 1, additive model in Example 2, multiple response regression in Example 3, balanced and unbalanced categorical response regression in Example 4, interaction affect regression in Example 5 and hetero-scedastic model in Example 6. We evaluate the performance through three criteria:

1. S: the minimum model size to include all active covariate variables. We report the $5 \%, 25 \%, 50 \%, 75 \%$, and $95 \%$ quantiles of $\mathrm{S}$ out of 500 replications.

2. Ps the proportion that an individual active covariate variable is selected for a given model size $\mathrm{d}$ in the 500 replications.
3. Pa the proportion that all active covariate variables are selected for a given model size $\mathrm{d}$ in the 500 replications.

Example 1: This example is designed to compare the finite sample performance of the DC-SIS with SIS (Fan and Lv 2008), DC-SIS (Li et al.), MDC-SIS [9] and SIRS [16]. In this example, we consider the linear model:

\section{$\mathrm{Y}=5 \mathrm{X} 1+5 \mathrm{X} 2+5 \mathrm{X} 3+10 \mathrm{X} 4+10 \mathrm{X} 5+\varepsilon$.}

where $\mathrm{X}$ are generated as follows: $\mathrm{Z} \sim \mathrm{N}(0, \mathrm{Ip})$,

$$
\begin{aligned}
& X_{k} \sim \text { Binomial }\left(\frac{\exp \left(Z_{i}\right)}{1+\exp \left(Z_{k}\right)}\right), i=4,6,8 \\
& X_{k} \sim \operatorname{Binomial}\left(\phi\left(Z_{k}\right)\right), k=5,7,9 \\
& X_{k}=Z_{k}, \text { others. }
\end{aligned}
$$
be 100 .

$\varepsilon$ is generated from the standard normal distribution. We set $\mathrm{n}$ to

Tables 1 and 2 summarize the simulation results of Example 1 . DWV-SIS is comparable with SIS and MDC-SIS, and performs better than DC-SIS and SIRS.

Example 2: In the example, we investigate the performance for additive models. As in Shao and Zhang [9], we consider the following two cases:

$$
\begin{array}{ll}
\text { Case 2a: } & Y=5 g_{1}\left(X_{1}\right)+3 g_{2}\left(X_{2}\right)+4 g_{3}\left(X_{3}\right)+6 g_{4}\left(X_{4}\right)+\sqrt{1.74_{\varepsilon}} \\
\text { Case 2b: } & \begin{array}{l}
Y=g_{1}\left(X_{1}\right)+g_{2}\left(X_{2}\right)+g_{3}\left(X_{3}\right)+g_{4}\left(X_{4}\right)+1.5 g_{1}\left(X_{5}\right)+1.5 g_{2}\left(X_{6}\right)+1.5 g_{3}\left(X_{7}\right)+ \\
1.5 g_{4}\left(X_{8}\right)+2 g_{1}\left(X_{9}\right)+2 g_{2}\left(X_{10}\right)+2 g_{3}\left(X_{11}\right)+2 g_{4}\left(X_{12}\right)+\sqrt{0.5184_{\varepsilon}}
\end{array}
\end{array}
$$

where $\mathrm{X} 1 ; \mathrm{X} 2 ;:::$; Xp are iid $\mathrm{U}(0 ; 1) . \varepsilon$ is generated from the standard normal distribution. As same as the Example 2 in Shao and Zhang [9], let $\mathrm{n}=400, \mathrm{~g} 1(\mathrm{x})=\mathrm{x}, \mathrm{g} 2(\mathrm{x})=(2 \mathrm{x}-1)^{2} \quad g_{3}(x)=\frac{\sin (2 \pi x)}{2-\sin (2 \pi x)}$ and $\mathrm{g}_{4}(x)=0.1$ $\sin (2 \pi x)+0.2 \cos (2 \pi x)+0.3 \sin ^{2}(2 \pi x)$

Tables 3-5 present the simulation results. We can find that MWVSIS is superior to SIS, NIS, MDC-SIS, DC-SIS and SIRS.

Example 3: In the example, we consider the following multiple response regression:

\section{$\mathrm{Y} 1=2 \mathrm{X} 1+2 \mathrm{X} 2+2 \mathrm{X} 3+2 \mathrm{X} 4+2 \mathrm{X} 5+\varepsilon 1$}

$\mathrm{Y} 2=2 \mathrm{X} 6+2 \mathrm{X} 7+2 \mathrm{X} 8+2 \mathrm{X} 9+2 \mathrm{X} 10+\varepsilon 2$

\begin{tabular}{|c|c|c|c|c|c|}
\hline Method & $\mathbf{5 \%}$ & $\mathbf{2 5 \%}$ & $\mathbf{5 0} \%$ & $\mathbf{7 5 \%}$ & $\mathbf{9 5 \%}$ \\
\hline SIS & 5.00 & 5.00 & 5.00 & 7.00 & 19.00 \\
\hline DWV-SIS & 5.00 & 5.00 & 6.00 & 7.00 & 19.00 \\
\hline MDC-SIS & 5.00 & 5.00 & 5.00 & 8.00 & 21.10 \\
\hline DC-SIS & 5.00 & 5.00 & 6.00 & 11.00 & 49.10 \\
\hline SIRS & 5.00 & 5.00 & 6.00 & 10.00 & 39.15 \\
\hline
\end{tabular}

Table 1: The $5 \%, 50 \%, 75 \%$ and $95 \%$ quantiles of $S$ in Example 1 .

\begin{tabular}{|c|c|c|c|c|c|c|}
\hline \multirow{2}{*}{ Method } & \multicolumn{5}{|c|}{$\mathbf{P}_{\mathbf{S}}$} & $\mathbf{P a}_{\mathbf{a}}$ \\
\cline { 2 - 7 } & $\mathbf{X}_{\mathbf{1}}$ & $\mathbf{X}_{\mathbf{2}}$ & $\mathbf{X}_{\mathbf{3}}$ & $\mathbf{X}_{\mathbf{4}}$ & $\mathbf{X}_{\mathbf{5}}$ & All \\
\hline SIS & 0.980 & 0.992 & 0.988 & 1.000 & 0.996 & 0.956 \\
\hline DWV-SIS & 0.986 & 0.986 & 0.988 & 1.000 & 1.000 & 0.960 \\
\hline MDC-SIS & 0.988 & 0.978 & 0.990 & 0.998 & 0.996 & 0.950 \\
\hline DC-SIS & 0.964 & 0.984 & 0.984 & 0.978 & 0.968 & 0.878 \\
\hline SIRS & 0.986 & 0.976 & 0.982 & 0.976 & 0.988 & 0.908 \\
\hline
\end{tabular}

Table 2: The proportion of $P_{S}$ and $P_{a}$ in Example 1. 


\begin{tabular}{|c|c|c|c|c|c|c|}
\hline Case & Method & $\mathbf{5 \%}$ & $\mathbf{2 5 \%}$ & $\mathbf{5 0 \%}$ & $\mathbf{7 5 \%}$ & $\mathbf{9 5 \%}$ \\
\hline \multirow{4}{*}{ a } & SIS & 49.80 & 262.00 & 489.00 & 753.75 & 950.15 \\
\cline { 2 - 7 } & NIS & 12.00 & 213.75 & 503.00 & 790.25 & 974.15 \\
\cline { 2 - 7 } & DWV-SIS & 4.00 & 4.00 & 4.00 & 5.00 & 18.00 \\
\cline { 2 - 7 } & MDC-SIS & 5.95 & 15.00 & 31.00 & 64.00 & 200.20 \\
\cline { 2 - 7 } & DC-SIS & 6.00 & 19.00 & 43.00 & 94.00 & 270.75 \\
\cline { 2 - 7 } & SIRS & 52.90 & 252.75 & 524.00 & 762.25 & 947.20 \\
\hline \multirow{4}{*}{ b } & SIS & 413.60 & 642.75 & 799.00 & 921.00 & 983.05 \\
\cline { 2 - 7 } & NIS & 632.90 & 845.25 & 937.50 & 981.00 & 999.00 \\
\cline { 2 - 7 } & DWV-SIS & 20.00 & 50.00 & 109.00 & 256.00 & 648.55 \\
\cline { 2 - 7 } & MDC-SIS & 45.90 & 104.50 & 184.00 & 322.00 & 698.05 \\
\cline { 2 - 7 } & DC-SIS & 48.00 & 116.00 & 224.50 & 391.75 & 758.15 \\
\cline { 2 - 7 } & SIRS & 447.90 & 671.00 & 819.00 & 912.25 & 976.05 \\
\hline
\end{tabular}

Table 3: The $5 \%, 50 \%, 75 \%$ and $95 \%$ quantiles of $S$ in Example 2 .

\begin{tabular}{c|c|c|c|c|c|}
\multirow{2}{*}{ Method } & \multicolumn{2}{|c|}{ Ps } & & Pa \\
\cline { 2 - 6 } & $\mathbf{X}_{1}$ & $\mathbf{X}_{2}$ & $\mathbf{X}_{\mathbf{3}}$ & $\mathbf{X}_{\mathbf{4}}$ & All \\
\hline SIS & 1 & 0.064 & 1 & 1 & 0.064 \\
\hline NIS & 1 & 0.132 & 1 & 1 & 0.132 \\
\hline DWV-SIS & 1 & 0.996 & 1 & 1 & 0.996 \\
\hline MDC-SIS & 1 & 0.758 & 1 & 1 & 0.758 \\
\hline DC-SIS & 1 & 0.640 & 1 & 1 & 0.640 \\
\hline SIRS & 1 & 0.070 & 1 & 1 & 0.070 \\
\hline
\end{tabular}

Table 4: The proportion of $\mathrm{P}_{\mathrm{S}}$ and $\mathrm{Pa}_{\mathrm{a}}$ in Case 2a of Example 2.

\begin{tabular}{|c|c|c|c|c|c|c|c|}
\hline \multirow[t]{2}{*}{ Method } & \multicolumn{6}{|c|}{$P_{s}$} & \multirow{2}{*}{$\begin{array}{l}\mathbf{P a} \\
\text { All }\end{array}$} \\
\hline & $x_{1}$ & $x_{2}$ & $x_{3}$ & $x_{4}$ & $x_{5}$ & $X_{6}$ & \\
\hline SIS & 0.786 & 0.070 & 0.878 & 0.618 & 0.988 & 0.064 & \\
\hline NIS & 0.318 & 0.022 & 0.454 & 0.284 & 0.630 & 0.026 & \\
\hline DWV-SIS & 0.752 & 0.576 & 0.978 & 0.890 & 0.974 & 0.950 & \\
\hline MDC-SIS & 0.778 & 0.278 & 0.944 & 0.948 & 0.984 & 0.678 & \\
\hline DC-SIS & 0.730 & 0.248 & 0.936 & 0.900 & 0.976 & 0.586 & \\
\hline SIRS & 0.73 & 0.064 & 0.854 & 0.548 & 0.978 & 0.064 & \\
\hline \multirow[t]{2}{*}{ SIS } & $x_{7}$ & $X_{8}$ & $X_{9}$ & $x_{10}$ & $x_{11}$ & $x_{12}$ & \\
\hline & 1 & 0.944 & 1 & 0.068 & 1 & 1 & \\
\hline NIS & 0.800 & 0.614 & 0.864 & 0.044 & 0.990 & 0.904 & 0.000 \\
\hline DWV-SIS & 1.000 & 0.996 & 1.000 & 1.000 & 1.000 & 1.000 & 0.328 \\
\hline MDC-SIS & 1 & 1 & 1.000 & 0.962 & 1 & 1 & 0.126 \\
\hline DC-SIS & 1 & 1 & 0.998 & 0.932 & 1 & 1 & 0.096 \\
\hline SIRS & 0.996 & 0.920 & 0.998 & 0.074 & 1 & 0.996 & 0.000 \\
\hline
\end{tabular}

Table 5: The proportion of $\mathrm{PS}_{\mathrm{S}}$ and $\mathrm{Pa}_{\mathrm{a}}$ in Case $2 \mathrm{~b}$ of Example 2.

where $\mathrm{X}$ are generated as follows: $\mathrm{Z} \sim \mathrm{N}(0, \mathrm{Ip})$,

$$
\begin{aligned}
& X_{k} \sim \text { Binomial }\left(\frac{\exp \left(Z_{i}\right)}{1+\exp \left(Z_{k}\right)}\right), i=4,8,11 \\
& X_{k} \sim \operatorname{Binomial}\left(\phi\left(Z_{k}\right)\right), k=5,7,12 \\
& X_{k}=Z_{k}, \text { others. }
\end{aligned}
$$
be 200 .

$\varepsilon$ is generated from the standard normal distribution. We set $\mathrm{n}$ to

Tables 6 and 7 show DWV-SIS is significantly better than DC-SIS.

Example 4: In the example, we investigate the performance for additive models. As in Cui et al. [10], we consider the following two cases:

Case 3a: balanced: $P \gamma(Y=\gamma)=\frac{1}{R}$

\begin{tabular}{|c|c|c|c|c|c|}
\hline Method & $\mathbf{5 \%}$ & $\mathbf{2 5 \%}$ & $\mathbf{5 0 \%}$ & $\mathbf{7 5 \%}$ & $\mathbf{9 5 \%}$ \\
\hline DWV-SIS & 10 & 10.00 & 10.00 & 11.00 & 12.00 \\
\hline DC-SIS & 14.00 & 44.75 & 164.00 & 486.00 & 992.05 \\
\hline
\end{tabular}

Table 6: The 5\%, 50\%, 75\% and 95\% quantiles of S in Example 3.

Case 3b: unbalanced: $P \gamma(Y=\gamma)=\frac{2[1+(\gamma+1 / R-1)]}{3 R}$

where $\mathrm{r}=1,2, \ldots, \mathrm{R}$. Given $\mathrm{Yk}=\mathrm{r}$, the ith covariate variable $\mathrm{Xk}$ is then generated by letting $X k=\mu r+\varepsilon i$, where the mean term $\mu \mathrm{r} \in \mathrm{R}^{\mathrm{R}}$ with $\mathrm{rth}$ component $\mu \mathrm{r}, \mathrm{r}=3$, but other components are all zero, and $\varepsilon \mathrm{i}=(\varepsilon i 1$, $\varepsilon i 2, \ldots, \varepsilon i p)^{\mathrm{T}}$ comes form $\mathrm{N}(0, \mathrm{Ip})$. Let $\mathrm{R}=10, \mathrm{n}=100$. In the example, we add MV-SIS because it a discriminant analysis screening with the categorical response variable.

Tables 8 and 9 show DWV-SIS slightly outperform than MV-SIS, and significantly better than DC-SIS.

Example 5. In the example, we consider the follow interaction item regression:

\section{$\mathrm{Y}=2 \mathrm{X} 1 \mathrm{X} 2+2 \mathrm{X} 3 \mathrm{X} 4+2 \mathrm{X} 5 \mathrm{X} 6+2 \mathrm{X} 7 \mathrm{X} 8+0.5 \varepsilon$}

where $\mathrm{X} 2, \mathrm{X} 4, \mathrm{X} 6, \mathrm{X} 8$ are iid $\mathrm{U}(0,1)$, other covariate variables are iid $\mathrm{t}(3)$. Because $\mathrm{X} 2, \mathrm{X} 4, \mathrm{X} 6, \mathrm{X} 8$ have no effect on mean of $\mathrm{Y}$, the mean screening methods do not distinguish these covariate variables, such as SIS, NIS and MDC-SIS. We only compare MWV-SIS with SISR and DC-SIS.

Tables 10 and 11 summarize the simulation results and show that the proposed DWV-SIS performs better than SIRS and DC-SIS.

Example 5: In the example, we consider the heteroscedastic model:

\section{$\mathrm{Y}=\mathrm{X} 1 \mathrm{X} 2+\mathrm{X} 3+\mathrm{X} 4+\mathrm{X} 5+\exp (\mathrm{X} 6+\mathrm{X} 7+\mathrm{X} 8) \varepsilon$}

where $\mathrm{Z}, \mathrm{X} 2, \mathrm{X} 3, \ldots, \mathrm{Xp}$ and $\varepsilon$ are iid $\mathrm{n}(0,1)$. $\mathrm{X} 1$ is generated form Poisson distribution with Z parameter. Like Example 5, X1, X6, X7, X8 have no effect on mean of $Y$. The sample size is 400 .

Form Tables 12 and 13, DWV-SIS performs comparable with DCSIR and better than SIRS [17-22].

\section{Real data analysis}

The Amazon commerce reviews can be found in https://archive.ics. uci.edu/ml/datasets/Amazon+Commerce+reviews+set, which is used for authorship identification in online Write print which is a new research field of pattern recognition. The data are derived from the customers reviews in Amazon Commerce Website for authorship identification, which collected 30 reviews for 50 of the most active users (represented by a unique ID and username). Based on lexical, content specific and idiosyncratic aspects, 10000 characteristic variables are collected, such as usage of digit, punctuation, words and sentences' length and usage frequency of words and so on. Compared with the sample size $n=1500$, the dimension $\mathrm{p}=10000$ is very large.

The response is a categorical variable. We use $\mathrm{R}$ function unique to extract unique elements for each covariate variable. From Table 14, the number of covariates which only include two elements are 2178. The maximum of unique elements is 297 . Hence, covariate variables can be viewed as categorical variables. DWV-SIS, MV-SIS and DC-SIS rank ten characteristic variables in Table 15. To compare the performance of three methods, we fit linear discriminant analysis using $\mathrm{R}$ function lda in MASS package. MV-SIS is the best with the total accuracy of $32.5 \%$ and DMV-SIS outperforms DC-SIS. 
Citation: Zhou J, Chen Y (2018) Ultra-high Dimensional Variable Screening via Density Weighted Variance. J Biom Biostat 9: 401. doi: 10.4172/21556180.1000401

Page 6 of 8

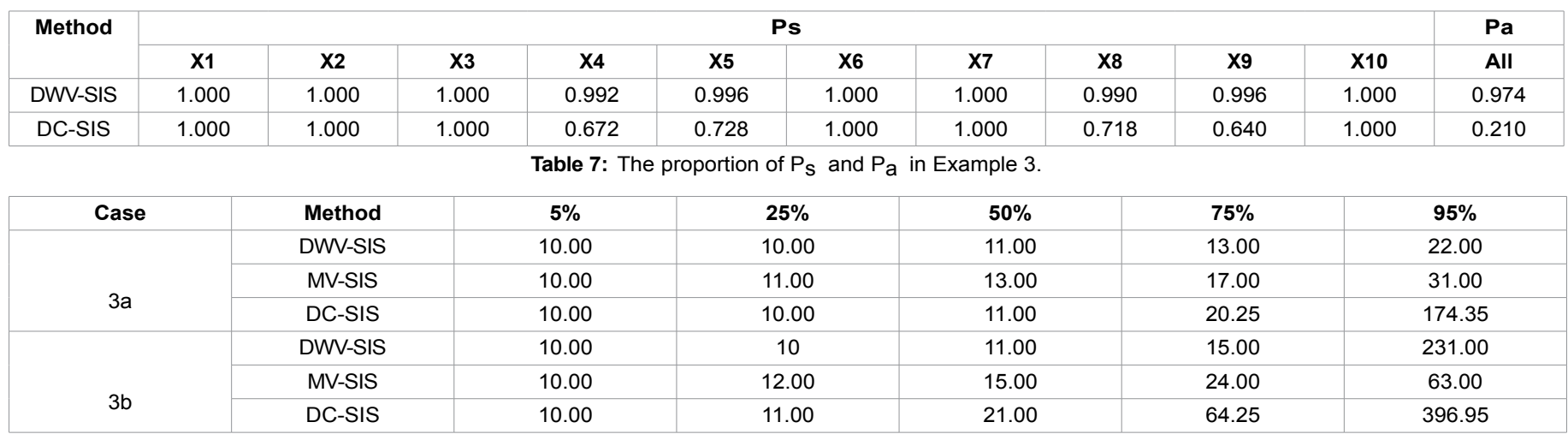

Table 8: The proportion of $\mathrm{P}_{\mathrm{S}}$ and $\mathrm{P}_{\mathrm{a}}$ in Example 4.

\begin{tabular}{|c|c|c|c|c|c|c|c|c|c|c|c|c|}
\hline Case & Method & \multicolumn{10}{|c|}{ Ps } & $\mathrm{Pa}$ \\
\hline \multirow{4}{*}{$3 a$} & & $\mathrm{X} 1$ & $\mathrm{X} 2$ & $\mathrm{X} 3$ & $X 4$ & X5 & $\times 6$ & $\mathbf{X 7}$ & X8 & X9 & $\mathbf{X 1 0}$ & All \\
\hline & DWV-SIS & 0.992 & 0.998 & 0.994 & 0.988 & 1.000 & 0.992 & 0.990 & 0.992 & 0.998 & 0.994 & 0.940 \\
\hline & MV-SIS & 0.980 & 0.972 & 0.980 & 0.978 & 0.980 & 0.982 & 0.976 & 0.990 & 0.992 & 0.988 & 0.866 \\
\hline & DC-SIS & 0.966 & 0.976 & 0.984 & 0.966 & 0.976 & 0.982 & 0.978 & 0.972 & 0.954 & 0.972 & 0.758 \\
\hline \multirow[b]{3}{*}{$3 b$} & DWV-SIS & 0.932 & 0.966 & 0.982 & 0.990 & 0.988 & 1.000 & 1.000 & 0.996 & 0.998 & 1.000 & 0.854 \\
\hline & MV-SIS & 0.930 & 0.930 & 0.948 & 0.936 & 0.946 & 0.952 & 0.934 & 0.952 & 0.948 & 0.946 & 0.700 \\
\hline & DC-SIS & 0.792 & 0.862 & 0.882 & 0.952 & 0.952 & 0.968 & 0.988 & 0.996 & 0.998 & 0.994 & 0.502 \\
\hline
\end{tabular}

Table 9: The proportion of $\mathrm{P}_{\mathrm{S}}$ and $\mathrm{Pa}_{\mathrm{a}}$ in Example 4.

\begin{tabular}{|c|c|c|c|c|}
\hline Method & $\mathbf{5 \%}$ & $\mathbf{2 5 \%}$ & $\mathbf{5 0 \%}$ & $\mathbf{9 5 \%}$ \\
\hline DWV-SIS & 8.00 & 8.00 & 9.00 & 16.00 \\
\hline SIRS & 136.95 & 273.00 & $408 \mathrm{v}$ & 606.5 \\
\hline DC-SIS & 499.75 & 786.75 & 942.00 & 838.75 \\
\hline
\end{tabular}

Table 10: The 5\%, 50\%, 75\% and 95\% quantiles of $S$ in Example 5.

\begin{tabular}{|c|c|c|c|c|c|c|c|c|c|}
\hline \multirow[t]{2}{*}{ Method } & \multicolumn{8}{|c|}{$P_{s}$} & \multirow{2}{*}{$\begin{array}{l}P_{a} \\
\text { All }\end{array}$} \\
\hline & $x_{1}$ & $x_{2}$ & $x_{3}$ & $x_{4}$ & $X_{5}$ & $x_{6}$ & $x_{7}$ & $x_{8}$ & \\
\hline DWV-SIS & 1.000 & 0.994 & 1.000 & 0.990 & 1.000 & 0.978 & 1.000 & 0.988 & 0.95 \\
\hline SIRS & 1.000 & 0.244 & 1.000 & 0.250 & 1.000 & 0.256 & 1.000 & 0.196 & 0.002 \\
\hline DC-SIS & 1.000 & 0.086 & 1.000 & 0.070 & 1.000 & 0.070 & 1.000 & 0.076 & 0 \\
\hline
\end{tabular}

Table 11: The proportion of $P_{S}$ and $P_{a}$ in Example 5.

\begin{tabular}{|c|c|c|c|c|}
\hline Method & $\mathbf{5 \%}$ & $\mathbf{2 5 \%}$ & $\mathbf{5 0 \%}$ & $\mathbf{9 5 \%}$ \\
\hline DWV-SIS & 8.00 & 9.00 & 12.00 & 15.00 \\
\hline SIRS & 15.00 & 27.00 & 42.00 & 70.00 \\
\hline DC-SIS & 8.00 & 8.00 & 8.00 & 136.45 \\
\hline
\end{tabular}

Table 12: The 5\%, 50\%, 75\% and 95\% quantiles of S in Example 6.

\begin{tabular}{|c|c|c|c|c|c|c|c|c|}
\hline \multirow{2}{*}{ Method } & \multicolumn{9}{|c|}{ Ps } & \multicolumn{2}{|c|}{ Pa } \\
\cline { 2 - 9 } & $\mathbf{X 1}$ & $\mathbf{X 2}$ & $\mathbf{X 3}$ & $\mathbf{X 4}$ & $\mathbf{X 5}$ & $\mathbf{X 6}$ & $\mathbf{X 7}$ \\
\hline DWV-SIS & 1.000 & 1.000 & 1.000 & 1.000 & 1.000 & 0.994 & 0.996 & 0.996 \\
\hline SIRS & 0.966 & 1.000 & 1.000 & 1.000 & 1.000 & 0.928 & 0.912 & 0.904 \\
\hline DC-SIS & 0.992 & 0.998 & 1.000 & 0.998 & 1.000 & 0.998 & 1.000 & 1.000 \\
\hline
\end{tabular}

Table 13: The proportion of $P_{S}$ and $P_{a}$ in Example 6.

\begin{tabular}{|c|c|c|c|c|}
\hline $\mathbf{2}$ & $\mathbf{4 1 0}$ & $\mathbf{4 5 0}$ & $\mathbf{5 1 0 0}$ & $\mathbf{5 1 5 0}$ \\
\hline $\mathbf{2 1 7 8}$ & 9339 & 9984 & 9996 & $\mathbf{9 9 9}$ \\
\hline
\end{tabular}

Table 14: The distribution of unique elements of $X$. 
Citation: Zhou J, Chen Y (2018) Ultra-high Dimensional Variable Screening via Density Weighted Variance. J Biom Biostat 9: 401. doi: 10.4172/21556180.1000401

Page 7 of 8

\begin{tabular}{|c|c|c|c|c|c|c|}
\hline \multirow{2}{*}{$\begin{array}{c}\text { Rank } \\
1-2\end{array}$} & \multicolumn{2}{|c|}{ DWV-SIS } & \multicolumn{2}{|c|}{ MV-SIS } & \multicolumn{2}{|c|}{ DC-SIS } \\
\hline & 6939 & 8058 & 6939 & 7469 & 6924 & 6939 \\
\hline $3-4$ & 6924 & 7468 & 6924 & 6629 & 6966 & 7009 \\
\hline $5-6$ & 7700 & 7009 & 8058 & 7700 & 6977 & 6955 \\
\hline $7-8$ & 7024 & 6740 & 6740 & 7009 & 6985 & 7014 \\
\hline $9-10$ & 6995 & 7011 & 6995 & 7024 & 7011 & 6969 \\
\hline Total Accuracy & \multicolumn{2}{|c|}{$25.47 \%$} & \multicolumn{2}{|c|}{$32.40 \%$} & \multicolumn{2}{|c|}{$20.26 \%$} \\
\hline
\end{tabular}

Table 15: Compared with some models of Amazon commerce reviews.

\section{References}

1. Fan J, Lv J (2010) A selective overview of variable selection in high dimensional feature space. Statistica Sinica 20: 101.

2. Fan J, Lv J (2008) Sure independence screening for ultrahigh dimensional feature space. JR Stat Soc Series B Stat Methodol 70: 849-911.

3. Wang $H$ (2009) Forward regression for ultra-high dimensional variable screening. J Am Stat Assoc 104: 1512-1524.

4. Fan J, Song R (2010) Sure independence screening in generalized linear models with NP-dimensionality. Ann Stat 38: 3567-3604.

5. Fan J, Feng $Y$, Song $R$ (2011) Nonparametric independence screening in sparse ultra-high-dimensional additive models. J Am Stat Assoc 106: 544-557.

6. Li G, Peng H, Zhang J, Zhu L (2012) Robust rank correlation based screening. Ann Stat 40: 1846-1877.

7. Szekely GJ, Rizzo ML, Bakirov NK (2007) Measuring and testing dependence by correlation of distances. Ann Stat 35: 2769-2794.

8. Li R, Zhong W, Zhu L (2012) Feature screening via distance correlation learning. J Am Stat Assoc 107: 1129-1139.

9. Shao X, Zhang J (2014) Martingale difference correlation and its use in highdimensional variable screening. J Am Stat Assoc 109: 1302-1318.

10. Cui H, Li R, Zhong W (2015) Model-free feature screening for ultrahigh dimensional discriminant analysis. J Am Stat Assoc 110: 630-641.

11. Zheng JX (1996) A consistent test of functional form via nonparametric estimation techniques. J Econom 75: 263-289.
12. Fan J, Samworth R, Wu Y (2009) Ultrahigh dimensional feature selection: beyond the linear model. J Mach Learn Res 10: 2013-2038.

13. Aitchison J, Aitken CG (1976) Multivariate binary discrimination by the kernel method. Biometrika 63: 413-420.

14. Hsiao C, Li Q, Racine JS (2007) A consistent model specification test with mixed discrete and continuous data. J Econom 140: 802-826.

15. Rajendra B (1997) Matrix Theory. Springer Verlag New York Inc.

16. Zhu LP, Li L, Li R, Zhu LX (2011) Model-free feature screening for ultrahighdimensional data. J Am Stat Assoc 106: 1464-1475.

17. Cho H, Fryzlewicz P (2012) High dimensional variable selection via tilting. JR Stat Soc Series B Stat Methodol 74: 593-622.

18. Fan J, Ma Y, Dai W (2014) Nonparametric independence screening in sparse ultra-high-dimensional varying coefficient models. J Am Stat Assoc 109: 1270 1284.

19. Hall $P$, Miller $H$ (2009) Using generalized correlation to effect variable selection in very high dimensional problems. J Comput Graph Stat 18: 533-550.

20. Liu J, Li R, Wu R (2014) Feature selection for varying coefficient models with ultrahigh-dimensional covariates. J Am Stat Assoc 109: 266-274.

21. Meier L, Van de Geer S, Bühlmann P (2009) High-dimensional additive modeling. Ann Stat 37: 3779-3821.

22. Serfling RJ (2009) Approximation theorems of mathematical statistics. John Wiley \& Sons 162 